\title{
Mathematical modeling of pulsatile blood flow and heat transfer under magnetic and vibrating environment
}

\author{
Ahmad R. Haghighi ${ }^{1,2 *}$, Nooshin Aliashrafi ${ }^{2}$ \\ ${ }^{1}$ Department of Mathematics, Technical and Vocational University, Tehran 17115-131, Iran \\ ${ }^{1,2}$ Department of Mathematics, Urmia University of Thechnology, Urmia 57155-419, Iran
}

Corresponding Author Email: ah.haghighi@gmail.com

https://doi.org/10.18280/ijht.360302

Received: 4 November 2017

Accepted: 5 May 2018

\section{Keywords:}

body acceleration, crank-Nicolson scheme, heat transfer, magnetic field, stenosis

\begin{abstract}
In the present study, a two-dimensional pulsatile blood flow model is created and the related heat transfer characteristics through a stenosed artery are investigated in the presence of a defined magnetic field with the body acceleration. The blood domain is assumed as a nonlinear, time-dependent, incompressible and laminar flow. The blood flow is considered with the unsteady characteristics because the pulsatile pressure gradient is arising due to the systematic reactions between the heart and the body acceleration. The non-linear momentum and continuity equations are solved with suitable initial and boundary conditions using the Crank-Nicolson scheme. In this study, the blood flow characteristics (velocity profiles, temperature, volumetric flow rate and flow resistance) are evaluated, also effects of the defined stenosis severity, the heat transfer factors and the considered magnetic field on the effective flow properties are discussed. Besides, the blood flow characteristics have been analyzed in a comparison form for two rigid and elastic arteries. Finally, it should be said that the present outputs are in good agreement with some available and validated results.
\end{abstract}

\section{INTRODUCTION}

The cardiovascular diseases are responsible for over 17.3 million deaths per year and the main causes of death in many countries [1-2]. Amongst the different cardiovascular diseases, the arteriosclerosis is the main one which affects the blood flow in the arteries [3]. In atherosclerosis, the fat particles and cholesterol are gatherd inside the blood arteries. This event causes the partial occlusion and reduces the blood transport in the area beyond the narrowing [1]. Depending on the stenosis artery's location, the atherosclerosis may lead to stroke, heart attack, and various cardiovascular diseases. Many researchers [4-6] have noted that the initiation and development of atherosclerosis are strongly related to the blood flow characteristics, therefore to study the effect of stenosed on blood flow characteristics through vessels, many theoretical and numerical investigations [2,7-10] have been performed.

Blood is a suspension of different cells such as red and white blood cells, leukocytes, and platelets in a special liquid called plasma [11-13]. Chakravarty and Mandal [14] took blood flow as a non-linear and incompressible flow through a stenosed artery and investigated the blood flow characteristics considering the mentioned conditions. They noted that assumption of the vessels rigidity isn't acceptable, so the vessels is considered to be an elastic domain and the stenosis's geometries are considered as a time-dependent boundaries. Lorenzini and Casalena [9] investigated CFD analysis of pulsatile blood flow in an atherosclerotic human artery with eccentric plaques. Marques et al. [10] investigated the pulsatile blood flow in a human artery which the vessel was assumed as a straight wall, also the blood flow was considered to be incompressible and axisymmetric. Also, the effect of pulsatile flow was taken into account by imposing the velocity profile of the cardiac cycle. Ponalagusmy and Selvi [15] have developed a mathematical model for blood flow through arterial stenosis with the two-fluid model including a core region of Casson fluid and a peripheral layer of Newtonian fluid. They concluded that the downstream of the stenotic regions is more important for the diagnosis of vessel diseases. Haghighi et al. [16] have studied a two-dimensional, pulsatile and two-layered blood flow through a tapered flexible artery. They solved the governing equations using the finite difference scheme and studied the effect of several factors such as the vessel tapering, the presence of stenosis and the wall motion on blood flow characteristics.

The magnetic field has an important application as a blood flow controller during surgery. In this regard, Misra et al. [1719] studied the varieties in blood behavior through arteries by operating Newtonian/ non-Newtonian fluid in the presence of a magnetic field. Mekheimer et al. [20] designed a mathematical model for blood flow through an elastic vessel having many stenoses in the presence of magnetic field. Shit [21] developed a computational model for blood flow in the presence of magnetic field. In this model he reported that no clinical disorders are observed for human health when a magnetic field is used up to $9 \mathrm{~T}$. Alshare et al. [22] tried to perform the flow simulations on the blood flow in an axisymmetric arterial stenosis using the non-Newtonian fluid model in the presence of magnetic field.

The flow of blood under the influence of body acceleration is significantly affected, while driving a vehicle or flying in an aircraft, because the blood flow may create serious health problems such as loss of vision, headache, increase of pulse rate and hemorrhage in face, neck and brain in a vibrating environment. Sankar and Lee [23] studied on the pulsatile flow of blood through an arterial stenosis considering blood as a 
core region of the Casson fluid and a peripheral layer of Newtonian fluid under periodic body acceleration. Also Shit and Roy [24] examined the pulsatile flow of blood through a stenosed artery under the influence of periodic body acceleration and then analyzed the heat transfer phenomena with constant blood viscosity.

Haghighi and Asl [25] solved a two-dimensional and pulsatile model of blood flow through a tapered artery with overlapping stenosis using the explicit scheme. The aim of the present study is investigation on blood flow in the presence of magnetic field and body acceleration. The governing equations have been solved using finite difference CrankNicolson scheme. This study tries to examine the effect of several factors such as the magnetic field, the presence of stenosis and the body acceleration on the heat transfer characteristics of the considered blood flow.

\section{THE GEOMETRY OF THE STENOSIS}

This numerical modeling considers a two-dimensional, laminar, unsteady, fully developed and axially symmetric flow of blood through a stenosed artery. Let $(r, \theta, z)$ is the cylindrical polar coordinates system in which $\mathrm{z}$ is taken along the axial direction and $\mathrm{r}, \theta$ are taken along the radial and circumferential directions, respectively. The geometry of the stenosis varies with time and is constructed mathematically as: (see Fig 1) [26-28].

$\mathrm{R}(\mathrm{z}, \mathrm{t})=\left\{\begin{array}{lc}1-\mathrm{A}\left[\mathrm{1}_{0}^{\mathrm{n}-1}(\mathrm{z}-\mathrm{d})-(\mathrm{z}-\mathrm{d})^{\mathrm{n}}\right] \mathrm{a}_{1}(\mathrm{t}), & \mathrm{d} \leq \mathrm{z} \leq \mathrm{d}+\mathrm{l}_{0} . \\ \mathrm{a}_{1}(\mathrm{t}), & \text { otherwise }\end{array}\right.$

And:

$$
\mathrm{A}=\frac{\delta}{\mathrm{R}_{0} \mathrm{~L}_{0}^{\mathrm{n}}} \frac{\mathrm{n}^{\mathrm{n}(\mathrm{n}-1)}}{(\mathrm{n}-1)}
$$

where, $\mathrm{R}(\mathrm{z}, \mathrm{t}), \mathrm{R}_{0}, \mathrm{~L}, \mathrm{l}_{0}, \mathrm{~d}$ and $\tau_{\mathrm{m}}$ are the radius of the arterial segment in the constricted region, the radius of the no stenotic artery, the finite length of the arterial segment, the length of the stenosis, the upstream length of the artery and the critical height of the stenosis, respectively. In the above equations, $\mathrm{n} \geq 2$ is the parameter representing the asymmetry of the stenosis, where $n=2$ represents that the stenosis is symmetric. The variant time parameter $\mathrm{a}_{1}(\mathrm{t})$ is given by $\mathrm{a}_{1}(\mathrm{t})=1+\mathrm{k}_{\mathrm{r}} \cos (\mathrm{w} \mathrm{t}+\varphi)$. Also $\varphi$ and $\mathrm{k}_{\mathrm{r}}$ are the phase angle and the amplitude parameter, respectively.

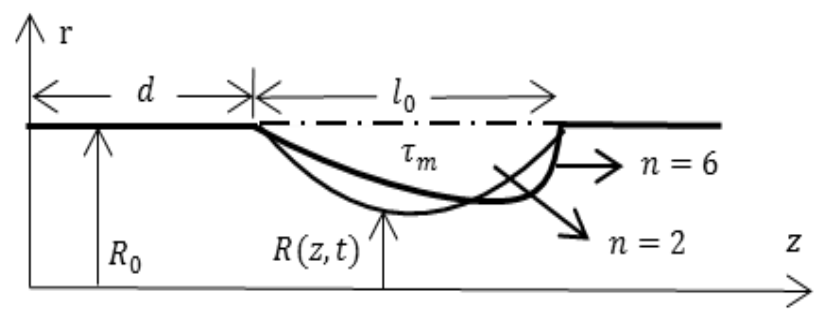

Figure 1. Schematic geometry of the stenosed artery

\section{THE GOVERNING EQUATIONS}

The governing equations for the blood flow in the cylindrical coordinate system can be written in nondimensional forms as follow [17, 25, 29]:

$$
\begin{aligned}
& \frac{\partial u}{\partial z}+\frac{v}{r}+\frac{\partial v}{\partial r}=0, \\
& \frac{\partial u}{\partial t}+u \frac{\partial u}{\partial z}+v \frac{\partial u}{\partial r}= \\
& \quad-\frac{\partial p}{\partial z}+\frac{1}{\alpha^{2}}\left(\frac{\partial^{2} u}{\partial r^{2}}+\frac{1}{r} \frac{\partial u}{\partial r}+\frac{\partial^{2} u}{\partial z^{2}}\right)-\frac{1}{\alpha^{2}}(h)^{2} u+\frac{1}{\alpha^{2}} G(t),
\end{aligned}
$$

$$
\begin{aligned}
\frac{\partial v}{\partial t}+ & u \frac{\partial v}{\partial z}+v \frac{\partial v}{\partial r} \\
& =-\frac{\partial p}{\partial r}+\frac{1}{\alpha^{2}}\left(\frac{\partial^{2} v}{\partial r^{2}}+\frac{1}{r} \frac{\partial v}{\partial r}+\frac{\partial^{2} v}{\partial z^{2}}-\frac{v}{r^{2}}\right) \\
\frac{\partial \theta}{\partial t}+ & u \frac{\partial \theta}{\partial z}+v \frac{\partial \theta}{\partial r} \\
& =\frac{1}{\alpha^{2} P_{r}}\left(\frac{\partial^{2} \theta}{\partial r^{2}}+\frac{1}{r} \frac{\partial \theta}{\partial r}+\frac{\partial^{2} \theta}{\partial z^{2}}\right)+\frac{E_{c}(h)^{2}}{\alpha^{2}} u^{2}
\end{aligned}
$$

The following non-dimensional variables are used in Eqs. $(2-5)$ :

$$
\begin{gathered}
\mathrm{u}=\frac{\mathrm{u}^{*}}{\mathrm{U}}, \mathrm{v}=\frac{\mathrm{v}^{*}}{\mathrm{U}}, \mathrm{r}=\frac{\mathrm{r}^{*}}{\mathrm{R}_{0}}, \mathrm{z}=\frac{\mathrm{z}^{*}}{\mathrm{R}_{0}}, \mathrm{t}=\frac{\mathrm{t}^{*} \mathrm{U}}{\mathrm{R}_{0}}, \mathrm{~d}=\frac{\mathrm{d}^{*}}{\mathrm{R}_{0}}, \mathrm{p}=\frac{\mathrm{p}^{*}}{\rho \mathrm{U}^{2}}, \\
\mathrm{l}_{0}=\frac{\mathrm{l}_{0}^{*}}{\mathrm{R}_{0}}, \mathrm{Re}=\frac{\rho \mathrm{UR}_{0}}{\mu}, \theta=\frac{\mathrm{T}^{*}-\mathrm{T}_{\infty}}{\mathrm{T}_{\mathrm{w}}-\mathrm{T}_{\infty}} .
\end{gathered}
$$

where, $\mathrm{u}$ and $\mathrm{v}$ are the axial and the radial dimensionless velocity components, respectively, $p$ is the pressure, $\rho$ is the density and $\mu$ is the viscosity of the fluid. The nondimensional parameters in Eqs. (2-5) are as follow, Womersley parameter $\alpha^{2}=\frac{\mathrm{UR}_{0}}{v}$, Hartmann number $\mathrm{h}=\mathrm{B}_{0} \mathrm{R}_{0} \sqrt{\frac{\sigma}{\rho v}}$, Prandtl number $\mathrm{P}_{\mathrm{r}}=\frac{\mu \mathrm{C}_{\mathrm{p}}}{\mathrm{K}}$ and Eckert number $\mathrm{E}_{\mathrm{c}}=\frac{\mathrm{U}^{2}}{\mathrm{C}_{\mathrm{p}}\left(\mathrm{T}_{\mathrm{W}}-\mathrm{T}_{\infty}\right)}[28-30]$.

The dimensionless pressure gradient $\frac{\partial p}{\partial z}$ in Eqs. (2-5) is assumed as:

$-\frac{\partial \mathrm{p}}{\partial \mathrm{z}}=\mathrm{A}_{0}+\mathrm{A}_{1} \cos \omega \mathrm{t}$

where,

a) $\mathrm{A}_{0}$ is the constant amplitude of the pressure gradient.

b) $A_{1}$ is the amplitude of the pulsatile component giving rise to the systolic and diastolic pressures.

c) $f_{p}$ is the pulse frequency. 
The body acceleration expression defined in the following form [28] using the non-dimensional quantities $\mathrm{a}_{0}=\frac{\mathrm{R}_{0}^{2} \mathrm{a}_{0}^{*}}{\mathrm{vU}}$ and $\mathrm{b}=\frac{\mathrm{w}_{\mathrm{b}}}{\mathrm{w}}$ :

$\mathrm{G}(\mathrm{t})=\mathrm{a}_{0} \cos \left(\mathrm{bt}+\varphi_{\mathrm{g}}\right)$.

The boundary conditions are considered as follows [31-33]:

$\begin{array}{llll}\mathrm{v}(\mathrm{r}, \mathrm{z}, \mathrm{t})=0, & \frac{\partial \mathrm{u}(\mathrm{r}, \mathrm{z}, \mathrm{t})}{\partial \mathrm{r}}=0, & \frac{\partial \theta(\mathrm{r}, \mathrm{z}, \mathrm{t})}{\partial \mathrm{r}}=0 & \mathrm{r}=0, \\ \mathrm{v}(\mathrm{r}, \mathrm{z}, \mathrm{t})=\frac{\partial \mathrm{R}}{\partial \mathrm{t}}, & \mathrm{u}(\mathrm{r}, \mathrm{z}, \mathrm{t})=0, & \theta(\mathrm{r}, \mathrm{z}, \mathrm{t})=1 & \mathrm{r}=\mathrm{R}(\mathrm{z}) .\end{array}$

In the transformed coordinate $\xi$, a radial coordinate transformation has been defined applying $\xi=\frac{\mathrm{r}}{\mathrm{R}}$ [8, 33-37]. In the following, Eqs. (2-5) and prescribed boundary conditions take the following forms using this transformation:

$\frac{\partial \mathrm{u}}{\partial \mathrm{z}}-\frac{\xi}{\mathrm{R}} \frac{\partial \mathrm{u}}{\partial \xi} \frac{\partial \mathrm{R}}{\partial \mathrm{z}}+\frac{\mathrm{v}}{\xi \mathrm{R}}+\frac{1}{\mathrm{R}} \frac{\partial \mathrm{v}}{\partial \xi}=0$

$$
\begin{aligned}
\frac{\partial \mathrm{u}}{\partial \mathrm{t}}= & -\frac{\partial \mathrm{p}}{\partial \mathrm{z}}+\frac{1}{\mathrm{R}} \frac{\partial \mathrm{u}}{\partial \xi}\left[\xi\left(\mathrm{u} \frac{\partial \mathrm{R}}{\partial \mathrm{z}}+\frac{\partial \mathrm{R}}{\partial \mathrm{t}}\right)-\mathrm{v}\right]-\mathrm{u} \frac{\partial \mathrm{u}}{\partial \mathrm{z}} \\
& +\frac{1}{\alpha^{2}}\left[\frac{1}{\mathrm{R}^{2}}\left\{1+\left(\xi \frac{\partial \mathrm{R}}{\partial \mathrm{z}}\right)^{2}\right\} \frac{\partial^{2} \mathrm{u}}{\partial \xi^{2}}\right. \\
& \left.+\frac{1}{\xi \mathrm{R}^{2}}\left\{1+2\left(\xi \frac{\partial \mathrm{R}}{\partial \mathrm{z}}\right)^{2}-\xi^{2} \mathrm{R} \frac{\partial^{2} \mathrm{R}}{\partial \mathrm{z}^{2}}\right\} \frac{\partial \mathrm{u}}{\partial \xi}+\frac{\partial^{2} \mathrm{u}}{\partial \mathrm{z}^{2}}\right] \\
& -\frac{1}{\alpha^{2}}(\mathrm{~h})^{2} \mathrm{u}+\frac{1}{\alpha^{2}} \mathrm{G}(\mathrm{t}),
\end{aligned}
$$$$
\frac{\partial \theta}{\partial \mathrm{t}}=\frac{1}{\mathrm{R}} \frac{\partial \theta}{\partial \xi}\left[\xi\left(\frac{\partial \mathrm{R}}{\partial \mathrm{t}}+\mathrm{u} \frac{\partial \mathrm{R}}{\partial \mathrm{z}}\right)-\mathrm{v}\right]
$$$$
-\mathrm{u} \frac{\partial \theta}{\partial \mathrm{z}}+\frac{1}{\alpha^{2} \mathrm{P}_{\mathrm{r}} \mathrm{R}^{2}}\left[\frac{\partial^{2} \theta}{\partial \xi^{2}}\left\{1+\left(\xi \frac{\partial \mathrm{R}}{\partial \mathrm{z}}\right)^{2}\right\}\right.
$$$$
+\frac{1}{\xi} \frac{\partial \theta}{\partial \xi}\left(1+2\left(\xi \frac{\partial \mathrm{R}}{\partial \mathrm{z}}\right)^{2}-\mathrm{R} \xi^{2} \frac{\partial^{2} \mathrm{R}}{\partial \mathrm{z}^{2}}\right)
$$$$
\left.+\mathrm{R}^{2} \frac{\partial^{2} \theta}{\partial \mathrm{z}^{2}}\right]+\frac{\mathrm{E}_{\mathrm{c}}(\mathrm{h})^{2}}{\alpha^{2}} \mathrm{u}^{2}
$$

$\mathrm{v}(\xi, \mathrm{z}, \mathrm{t})=0, \frac{\partial \mathrm{u}(\xi, \mathrm{z}, \mathrm{t})}{\partial \xi}=0, \frac{\partial \theta(\xi, \mathrm{z}, \mathrm{t})}{\partial \xi}=0 \quad \xi=0$,

$\mathrm{v}(\xi, \mathrm{z}, \mathrm{t})=\frac{\partial \mathrm{R}}{\partial \mathrm{t}}, \mathrm{u}(\xi, \mathrm{z}, \mathrm{t})=0, \quad \theta(\xi, \mathrm{z}, \mathrm{t})=0 \quad \xi=1$

\section{THE RADIAL VELOCITY COMPONENT}

After multiplying Eq. (8) by $\xi R$ and integrating with respect to $\xi$ form 0 to $\xi$, we get:

$\xi \mathrm{v}(\xi, \mathrm{z}, \mathrm{t})+\mathrm{R} \int_{0}^{\xi} \xi \frac{\partial \mathrm{u}}{\partial \mathrm{z}} \mathrm{d} \xi-\frac{\partial \mathrm{R}}{\partial \mathrm{z}} \xi^{2} \mathrm{u}+\frac{\partial \mathrm{R}}{\partial \mathrm{z}} \int_{0}^{\xi} 2 \xi \mathrm{ud} \xi=0$.

Also by using the boundary conditions (12) at $\xi=1$, Eq. (13) is obtained as:

$\int_{0}^{1} \xi \frac{\partial \mathrm{u}}{\partial \mathrm{z}} \mathrm{d} \xi=-\int_{0}^{1} \frac{2}{\mathrm{R}} \frac{\partial \mathrm{R}}{\partial \mathrm{z}} \xi \mathrm{ud} \xi+\int_{0}^{1} \frac{1}{\mathrm{R}}\left(\frac{\partial \mathrm{R}}{\partial \mathrm{t}} \xi \mathrm{f}(\xi)\right) \mathrm{d} \xi$.

where $\mathrm{f}(\xi)$ represents an arbitrary function satisfying $\int_{0}^{\xi} \xi \mathrm{f}(\xi) \mathrm{d} \xi=1$. Let choose $\mathrm{f}(\xi)=-4\left(1-\xi^{2}\right)$. Taking the approximation of treating the equality between the integrals to integrands, Eq. (13) can be written as:

$\frac{\partial \mathrm{u}}{\partial \mathrm{z}}=-\frac{2}{\mathrm{R}} \frac{\partial \mathrm{R}}{\partial \mathrm{z}} \mathrm{u}-\frac{4}{\mathrm{R}}\left(1-\xi^{2}\right) \frac{\partial \mathrm{R}}{\partial \mathrm{t}}$.

By substituting (15) into (13), the radial velocity component can be obtained as follows:

$\mathrm{v}(\xi, \mathrm{z}, \mathrm{t})=\xi\left[\frac{\partial \mathrm{R}}{\partial \mathrm{z}} \mathrm{u}+\frac{\partial \mathrm{R}}{\partial \mathrm{t}}\left(2-\xi^{2}\right)\right]$.

\section{COMPUTATIONAL SCHEME}

To solve Eqs. (9)-(10) using a Crank-Nicolson scheme, the central difference formula is used to express the spatial derivatives and the forward difference formula is applied to the time derivative as bellow:

$\frac{\partial \mathrm{u}}{\partial \xi}=\frac{1}{2}\left[\frac{(\mathrm{u})_{\mathrm{i}, \mathrm{j}+1}^{\mathrm{k}}-(\mathrm{u})_{\mathrm{i}, \mathrm{j}-1}^{\mathrm{k}}}{2 \Delta \xi}+\frac{(\mathrm{u})_{\mathrm{i}, \mathrm{j}+1}^{\mathrm{k}+1}-(\mathrm{u})_{\mathrm{i}, \mathrm{j}-1}^{\mathrm{k}+1}}{2 \Delta \xi}\right]$,

$\frac{\partial u}{\partial z}=\frac{(u)_{i+1, j}^{k}-(u)_{i-1, j}^{k}}{2 \Delta z}$,

$\frac{\partial^{2} \mathrm{u}}{\partial \xi^{2}}=\frac{1}{2}\left[\frac{(u)_{i, j+1}^{\mathrm{k}}-2(\mathrm{u})_{\mathrm{i}, \mathrm{j}}^{\mathrm{k}}+(\mathrm{u})_{\mathrm{i}, \mathrm{j}-1}^{\mathrm{k}}}{(\Delta \xi)^{2}}+\frac{(\mathrm{u})_{\mathrm{i}, \mathrm{j}+1}^{\mathrm{k}+1}-2(\mathrm{u})_{\mathrm{i}, \mathrm{j}}^{\mathrm{k}+1}+(\mathrm{u})_{\mathrm{i}, \mathrm{j}-1}^{\mathrm{k}+1}}{(\Delta \xi)^{2}}\right]$,

$\frac{\partial^{2} \mathrm{u}}{\partial \mathrm{z}^{2}}=\frac{(\mathrm{u})_{\mathrm{i}+1, j}^{\mathrm{k}}-2(\mathrm{u})_{\mathrm{i}, \mathrm{j}}^{\mathrm{k}}+(\mathrm{u})_{\mathrm{i}-1, \mathrm{j}}^{\mathrm{k}}}{(\Delta \mathrm{z})^{2}}$,

$\frac{\partial \mathrm{u}}{\partial \mathrm{t}}=\frac{(\mathrm{u})_{\mathrm{i}, \mathrm{j}}^{\mathrm{k}+1}-(\mathrm{u})_{\mathrm{i}, \mathrm{j}}^{\mathrm{k}}}{\Delta \mathrm{t}}$.

We define:

$\begin{cases}\xi_{\mathrm{j}}=(\mathrm{j}-1) \Delta \xi, & (\mathrm{j}=1,2, . ., \mathrm{N}+1), \\ \mathrm{z}_{\mathrm{i}}=(\mathrm{i}-1) \Delta \mathrm{z}, & (\mathrm{i}=1,2, . ., \mathrm{M}+1), \\ \mathrm{t}_{\mathrm{k}}=(\mathrm{k}-1) \Delta \mathrm{t}, & (\mathrm{i}=1,2, \ldots)\end{cases}$ 
where, $\Delta \xi, \Delta \mathrm{z}, \Delta \mathrm{t}$ are the increments in the radial, the axial and the time directions respectively. Using the CrankNicolson scheme, the discretized form of Eq. (9) is given as:

$$
A_{i, j} u_{i, j-1}^{k+1}+B_{i, j} u_{i, j}^{k+1}+C_{i, j} u_{i, j+1}^{k+1}=D_{i, j} \text {. }
$$

where

$$
\begin{aligned}
& A_{i, j}=\frac{\Delta t}{4 \Delta \xi R_{i}^{k}}\left[\xi_{j}\left(\left(\frac{\partial R}{\partial t}\right)_{i}^{k}+u_{i, j}\left(\frac{\partial R}{\partial z}\right)_{i}^{k}\right)-v_{i, j}\right] \\
& -\frac{\Delta \mathrm{t}}{2\left(\mathrm{R}_{\mathrm{i}}^{\mathrm{k}}\right)^{2} \alpha^{2} \Delta \xi^{2}}\left\{1+\left(\xi_{\mathrm{j}}\left(\frac{\partial \mathrm{R}}{\partial \mathrm{z}}\right)_{\mathrm{i}}^{\mathrm{k}}\right)^{2}\right\} \\
& +\frac{\Delta \mathrm{t}}{4 \alpha^{2}\left(\mathrm{R}_{\mathrm{i}}^{\mathrm{k}}\right)^{2} \xi_{\mathrm{j}} \Delta \xi}\left\{1+2\left(\xi_{\mathrm{j}}\left(\frac{\partial \mathrm{R}}{\partial \mathrm{z}}\right)_{\mathrm{i}}^{\mathrm{k}}\right)^{2}-\xi_{\mathrm{j}}^{2} \mathrm{R}_{\mathrm{i}}^{\mathrm{k}}\left(\frac{\partial^{2} \mathrm{R}}{\partial \mathrm{z}^{2}}\right)_{\mathrm{i}}^{\mathrm{k}}\right\}, \\
& \mathrm{B}_{\mathrm{i}, \mathrm{j}}=1+\frac{\Delta \mathrm{t}}{\alpha^{2}\left(\mathrm{R}_{\mathrm{i}}^{\mathrm{k}}\right)^{2} \Delta \xi^{2}}\left\{1+\left(\xi_{\mathrm{j}}\left(\frac{\partial \mathrm{R}}{\partial \mathrm{z}}\right)_{\mathrm{i}}^{\mathrm{k}}\right)^{2}\right\}, \\
& \mathrm{C}_{\mathrm{i}, \mathrm{j}}=\frac{-\Delta \mathrm{t}}{4 \Delta \xi \mathrm{R}_{\mathrm{i}}^{\mathrm{k}}}\left[\xi_{\mathrm{j}}\left(\left(\frac{\partial \mathrm{R}}{\partial \mathrm{t}}\right)_{\mathrm{i}}^{\mathrm{k}}+\mathrm{u}_{\mathrm{i}, \mathrm{j}}\left(\frac{\partial \mathrm{R}}{\partial \mathrm{z}}\right)_{\mathrm{i}}^{\mathrm{k}}\right)-\mathrm{v}_{\mathrm{i}, \mathrm{j}}\right] \\
& -\frac{\Delta \mathrm{t}}{2\left(\mathrm{R}_{\mathrm{i}}^{\mathrm{k}}\right)^{2} \alpha^{2} \Delta \xi^{2}}\left\{1+\left(\xi_{\mathrm{j}}\left(\frac{\partial \mathrm{R}}{\partial \mathrm{z}}\right)_{\mathrm{i}}^{\mathrm{k}}\right)^{2}\right\} \\
& -\frac{\Delta \mathrm{t}}{2 \alpha^{2}\left(\mathrm{R}_{\mathrm{i}}^{\mathrm{k}}\right)^{2} \xi_{\mathrm{j}} \Delta \xi}\left\{1+2\left(\xi_{\mathrm{j}}\left(\frac{\partial \mathrm{R}}{\partial \mathrm{z}}\right)_{\mathrm{i}}^{\mathrm{k}}\right)^{2}-\xi_{\mathrm{j}}^{2} \mathrm{R}_{\mathrm{i}}^{\mathrm{k}}\left(\frac{\partial^{2} \mathrm{R}}{\partial \mathrm{z}^{2}}\right)_{\mathrm{i}}^{\mathrm{k}}\right\} \text {, } \\
& D_{i, j}=u_{i, j}^{k}-\Delta t\left(\frac{\partial R}{\partial z}\right)+\Delta t\left(\frac{G(t)}{\alpha^{2}}\right)-\Delta t\left(\frac{h^{2}}{\alpha^{2}}\right) u_{i, j} \\
& +\frac{\Delta \mathrm{t}}{4 \mathrm{R}_{\mathrm{i}}^{\mathrm{k}} \Delta \xi}\left[\xi_{j}\left(\mathrm{u}_{\mathrm{i}, \mathrm{j}}\left(\frac{\partial \mathrm{R}}{\partial \mathrm{z}}\right)_{\mathrm{i}}^{\mathrm{k}}+\left(\frac{\partial \mathrm{R}}{\partial \mathrm{t}}\right)_{\mathrm{i}}^{\mathrm{k}}\right)-\mathrm{v}_{\mathrm{i}, \mathrm{j}}\right] \\
& \left((u)_{i, j+1}^{k}-(u)_{i, j-1}^{k}\right)-\frac{\Delta t}{2 \Delta z}(u)_{i, j}^{k}\left((u)_{i+1, j}^{k}-(u)_{i-1, j}^{k}\right) \\
& +\frac{\Delta t}{2 \alpha^{2}\left(R_{i}^{k}\right)^{2} \Delta \xi^{2}}\left((u)_{i, j+1}^{k}-2(u)_{i, j}^{k}+(u)_{i, j-1}^{k}\right) \\
& \left\{1+\left(\xi_{j}\left(\frac{\partial R}{\partial z}\right)_{i}^{k}\right)^{2}\right\}+\frac{\Delta t}{4 \alpha^{2}\left(R_{i}^{k}\right)^{2} \xi_{j} \Delta \xi} \\
& \left\{1+2\left(\xi_{j}\left(\frac{\partial R}{\partial z}\right)_{i}^{\mathrm{k}}\right)^{2}-\xi_{j}^{2} R_{i}^{\mathrm{k}}\left(\frac{\partial^{2} \mathrm{R}}{\partial \mathrm{z}^{2}}\right)_{\mathrm{i}}^{\mathrm{k}}\right\} \\
& \left((u)_{i, j+1}^{k}-(u)_{i, j-1}^{k}\right)+\frac{\Delta t}{\alpha^{2} \Delta z^{2}} \\
& \left((u)_{i+1, j}^{k}-2(u)_{i, j}^{k}+(u)_{i-1, j}^{k}\right) \text {. }
\end{aligned}
$$

And the discretized form of Eq. (10) is:

$P_{i, j} \theta_{i, j-1}^{k+1}+Q_{i, j} \theta_{i, j}^{k+1}+E_{i, j} \theta_{i, j+1}^{k+1}=S_{i, j}$ where

$$
\begin{aligned}
& P_{i, j}=\frac{\Delta t}{4 \Delta \xi R_{i}^{k}}\left[\xi_{j}\left(\left(\frac{\partial R}{\partial t}\right)_{i}^{k}+u_{i, j}\left(\frac{\partial R}{\partial z}\right)_{i}^{k}\right)-v_{i, j}\right] \\
& -\frac{\Delta \mathrm{t}}{2\left(\mathrm{R}_{\mathrm{i}}^{\mathrm{k}}\right)^{2} \alpha^{2} \mathrm{P}_{\mathrm{r}} \Delta \xi^{2}}\left\{1+\left(\xi_{\mathrm{j}}\left(\frac{\partial \mathrm{R}}{\partial \mathrm{z}}\right)_{\mathrm{i}}^{\mathrm{k}}\right)^{2}\right\} \\
& +\frac{\Delta \mathrm{t}}{4 \alpha^{2} \mathrm{P}_{\mathrm{r}}\left(\mathrm{R}_{\mathrm{i}}^{\mathrm{k}}\right)^{2} \xi_{\mathrm{j}} \Delta \xi}\left\{1+2\left(\xi_{\mathrm{j}}\left(\frac{\partial \mathrm{R}}{\partial \mathrm{z}}\right)_{\mathrm{i}}^{\mathrm{k}}\right)^{2}-\xi_{\mathrm{j}}^{2} \mathrm{R}_{\mathrm{i}}^{\mathrm{k}}\left(\frac{\partial^{2} \mathrm{R}}{\partial \mathrm{z}^{2}}\right)_{\mathrm{i}}^{\mathrm{k}}\right\} \text {, } \\
& \mathrm{Q}_{\mathrm{i}, \mathrm{j}}=1+\frac{\Delta \mathrm{t}}{\alpha^{2} \mathrm{P}_{\mathrm{r}}\left(\mathrm{R}_{\mathrm{i}}^{\mathrm{k}}\right)^{2} \Delta \xi^{2}}\left\{1+\left(\xi_{\mathrm{j}}\left(\frac{\partial \mathrm{R}}{\partial \mathrm{z}}\right)_{\mathrm{i}}^{\mathrm{k}}\right)^{2}\right\}, \\
& E_{i, j}=\frac{-\Delta t}{4 \Delta \xi R_{i}^{k}}\left[\xi_{j}\left(\left(\frac{\partial R}{\partial t}\right)_{i}^{k}+u_{i, j}\left(\frac{\partial R}{\partial z}\right)_{i}^{k}\right)-v_{i, j}\right] \\
& -\frac{\Delta \mathrm{t}}{2\left(\mathrm{R}_{\mathrm{i}}^{\mathrm{k}}\right)^{2} \alpha^{2} \mathrm{P}_{\mathrm{r}} \Delta \xi^{2}}\left\{1+\left(\xi_{\mathrm{j}}\left(\frac{\partial \mathrm{R}}{\partial \mathrm{z}}\right)_{\mathrm{i}}^{\mathrm{k}}\right)^{2}\right\} \\
& -\frac{\Delta \mathrm{t}}{4 \alpha^{2} \mathrm{P}_{\mathrm{r}}\left(\mathrm{R}_{\mathrm{i}}^{\mathrm{k}}\right)^{2} \xi_{\mathrm{j}} \Delta \xi}\left\{1+2\left(\xi_{\mathrm{j}}\left(\frac{\partial \mathrm{R}}{\partial \mathrm{z}}\right)_{\mathrm{i}}^{\mathrm{k}}\right)^{2}-\xi_{\mathrm{j}}^{2} \mathrm{R}_{\mathrm{i}}^{\mathrm{k}}\left(\frac{\partial^{2} \mathrm{R}}{\partial \mathrm{z}^{2}}\right)_{\mathrm{i}}^{\mathrm{k}}\right\}, \\
& S_{i, j}=\theta_{i, j}^{k}+\frac{\Delta t}{4 R_{i}^{k} \Delta \xi}\left((\theta)_{i, j+1}^{k}-(\theta)_{i, j-1}^{k}\right) \\
& {\left[\xi_{j}\left(\left(\frac{\partial R}{\partial t}\right)_{i}^{k}+u_{i, j}\left(\frac{\partial R}{\partial z}\right)_{i}^{k}\right)-v_{i, j}\right]} \\
& -\frac{\Delta \mathrm{t}}{2 \Delta \mathrm{z}} \mathrm{u}_{\mathrm{i}, \mathrm{j}}^{\mathrm{k}}\left((\theta)_{\mathrm{i}+1, \mathrm{j}}^{\mathrm{k}}-(\theta)_{\mathrm{i}-1, \mathrm{j}}^{\mathrm{k}}\right) \\
& +\frac{\Delta \mathrm{t}}{2 \alpha^{2} \mathrm{P}_{\mathrm{r}}\left(\mathrm{R}_{\mathrm{i}}^{\mathrm{k}}\right)^{2} \Delta \xi^{2}}\left\{1+\left(\xi_{\mathrm{j}}\left(\frac{\partial \mathrm{R}}{\partial \mathrm{z}}\right)_{\mathrm{i}}^{\mathrm{k}}\right)^{2}\right\} \\
& \left((\theta)_{\mathrm{i}, \mathrm{j}+1}^{\mathrm{k}}-2(\theta)_{\mathrm{i}, \mathrm{j}}^{\mathrm{k}}+(\theta)_{\mathrm{i}, \mathrm{j}-1}^{\mathrm{k}}\right) \\
& +\frac{\Delta \mathrm{t}}{4 \alpha^{2} \mathrm{P}_{\mathrm{r}}\left(\mathrm{R}_{\mathrm{i}}^{\mathrm{k}}\right)^{2} \xi_{\mathrm{j}} \Delta \xi^{2}}\left\{1+2\left(\xi_{\mathrm{j}}\left(\frac{\partial \mathrm{R}}{\partial \mathrm{z}}\right)_{\mathrm{i}}^{\mathrm{k}}\right)^{2}-\xi_{\mathrm{j}}^{2} \mathrm{R}_{\mathrm{i}}^{\mathrm{k}}\right. \\
& \left.\left(\frac{\partial^{2} \mathrm{R}}{\partial \mathrm{z}^{2}}\right)_{\mathrm{i}}^{\mathrm{k}}\right\}\left((\theta)_{\mathrm{i}, \mathrm{j}+1}^{\mathrm{k}}-(\theta)_{\mathrm{i}, \mathrm{j}-1}^{\mathrm{k}}\right) \\
& +\frac{\Delta \mathrm{t}}{\alpha^{2} \mathrm{P}_{\mathrm{r}} \Delta \mathrm{z}^{2}}\left((\theta)_{\mathrm{i}+1, \mathrm{j}}^{\mathrm{k}}-2(\theta)_{\mathrm{i}, \mathrm{j}}^{\mathrm{k}}+(\theta)_{\mathrm{i}-1, \mathrm{j}}^{\mathrm{k}}\right) \\
& +\frac{\Delta \mathrm{tE}_{\mathrm{c}}(\mathrm{h})^{2}}{\alpha^{2}}\left(\mathrm{u}_{\mathrm{i}, \mathrm{j}}^{\mathrm{k}}\right)^{2} \text {. }
\end{aligned}
$$

The volumetric flow rate $(\mathrm{Q})$, the resistive impedance $(\Lambda)$ and the Nusselt number $\left(\mathrm{N}_{\mathrm{u}}\right)$ can be obtained using the following formulas:

$\mathrm{Q}_{\mathrm{i}}^{\mathrm{k}}=2 \pi\left(\mathrm{R}_{\mathrm{i}}^{\mathrm{k}}\right)^{2} \int_{0}^{1} \xi_{\mathrm{j}}(\mathrm{u})_{\mathrm{i}, \mathrm{j}}^{\mathrm{k}} \mathrm{d} \xi_{\mathrm{j}}$. 


$$
\begin{aligned}
& \Lambda_{\mathrm{i}}^{\mathrm{k}}=\frac{\left(\left|\mathrm{L}\left(\frac{\partial \mathrm{p}}{\partial \mathrm{z}}\right)_{\mathrm{i}}^{\mathrm{k}}\right|\right)}{\mathrm{Q}_{\mathrm{i}}^{\mathrm{k}}} . \\
& \mathrm{Nu}_{\mathrm{i}}=\frac{1}{\mathrm{R}_{\mathrm{i}}^{\mathrm{k}}}\left(\frac{\left.\theta_{\mathrm{i}, \mathrm{n}}^{\mathrm{k}}-\theta_{\mathrm{i}, \mathrm{n}-1}^{\mathrm{k}}\right) .}{\Delta \xi} .\right.
\end{aligned}
$$

\section{NUMERICAL RESULTS AND DISCUSSION}

The numerical computations have been carried out using the following parameter values [14, 34, 38]:

$$
\begin{gathered}
\Delta \mathrm{t}=0.001, \Delta \xi=0.0125, \Delta \mathrm{z}=0.1, \mathrm{~d}=10, \alpha=4, \mathrm{f}_{\mathrm{p}}=1.2, \\
\mathrm{~A}_{0}=0.1, \mathrm{~A}_{1}=0.2 \mathrm{~A}_{0}, \mathrm{~L}=30, \mathrm{l}_{0}=14, \mathrm{R}_{0}=1.52, \\
\mathrm{k}_{\mathrm{r}}=0.05, \mathrm{a}_{0}=1, \varphi_{\mathrm{g}}=\pi / 4, \varphi=0, \mathrm{~b}=1
\end{gathered}
$$

In order to validate the proposed results, the obtained axial velocity for the blood flow through a stenosed artery in the maximum constricted region for $\mathrm{h}=0$ and at the time $\mathrm{t}=2$ is compared with the corresponding results obtained by Shit and Roy [24]. The present results are in good agreement with Shit and Roy's [24] results

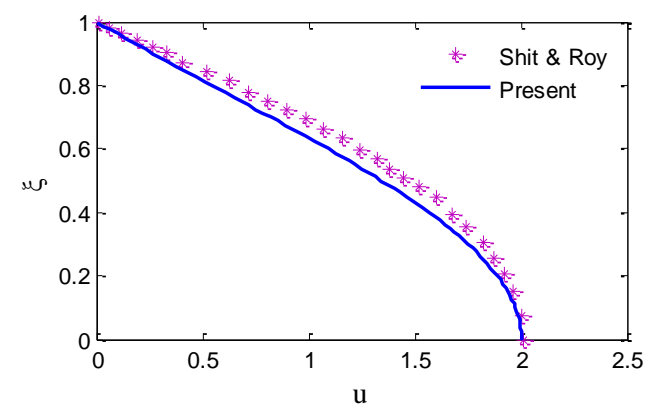

Figure 2. Comparison of the dimensionless axial velocity profile

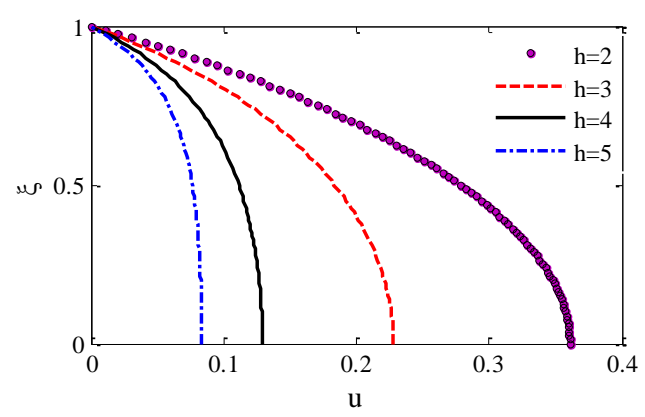

Figure 3. Dimensionless axial velocity profile for different values of Hartmann number

Fig. 3 shows the axial velocity profiles of the blood flow through the stenosed artery, at the specific conditions including; $\mathrm{z}=17, \mathrm{t}=4, \mathrm{n}=2$ and $\tau_{\mathrm{m}}=0.2 \mathrm{R}_{0}$, for different values of Hartmann number. Fig. 3 depicts that the axial velocity decreases with decrease in the Hartmann number. This event happens due to the interactions between the magnetic field and the blood flow. Furthermore, the axial velocity reaches to maximum value at the central line of the artery in all four cases.

Fig. 4 illustrates the dimensionless axial velocity profiles of the blood flow at $\mathrm{h}=2$ and $\mathrm{n}=2$ for different stenosis sizes. According to Fig 4, the axial velocity decreases by increasing the size of stenosis. The present figure includes the axial velocity at $\mathrm{t}=3$ through an elastic and rigid artery. The results show that the axial velocity in elastic artery is more than the axial velocity in the rigid artery. This difference between the axial velocities shows the importance of the assumption of elastic blood vessels.

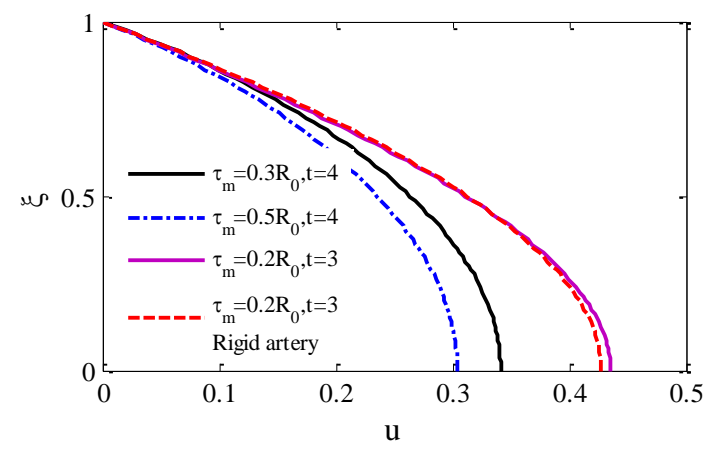

Figure 4. Dimensionless axial velocity profile for different stenosis sizes

The axial velocity variations are shown in Fig 5 for different amplitudes of body acceleration at $\mathrm{t}=4$ for $\tau_{\mathrm{m}}=0.2 \mathrm{R}_{0}, \mathrm{~h}=2$ and $n=2$. As another result, the axial velocity increases as the amplitude of body acceleration increases. Thus, in the presence of vibration, the environmental system may produce an increase in the blood velocity.

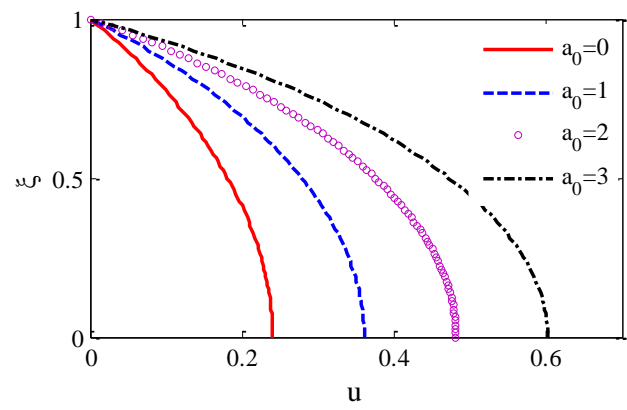

Figure 5. Dimensionless axial velocity profile for different amplitudes of body acceleration

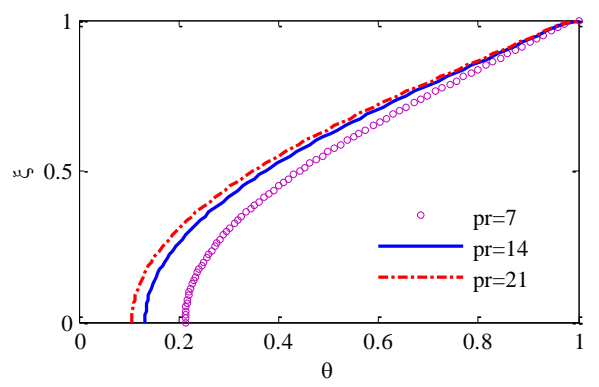

Figure 6. Distribution of the temperature profile for different values of Prandtl number 
Figs. 6 and 7 show the variation of heat transfer and Nusselt number for different Prandtl numbers. It is observed from Fig 6 that the temperature decreases with the increase of the Prandtl number. Fig 7 indicates that the Nusselt number increases with the increase of the Prandtl number. This variation of heat transfer and Nusselt number occurs for $h=2$, $\mathrm{n}=2$ and $\tau_{\mathrm{m}}=0.2 \mathrm{R}_{0}$ at $\mathrm{t}=4$. As seen in Fig. 7, when the Prandtl number increases, the thermal boundary layer thickness reduces. So, the less thermal conductivity leads to decrease in the heat conduction capacity.

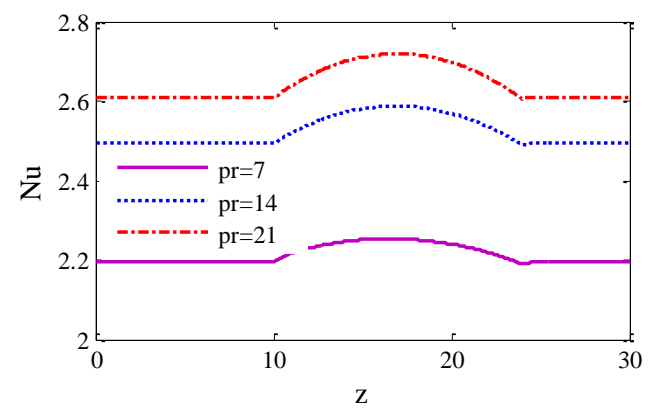

Figure 7. Distribution of the Nusselt number for different values of Prandtl number

The flow rate in the stenosed artery for different Hartmann numbers at $\mathrm{t}=4, \mathrm{n}=2$ and $\tau_{\mathrm{m}}=0.2 \mathrm{R}_{0}$ is shown in Fig 8 .

According to Fig 8, the flow rate decreases with the increase of the Hartmann number. As a consequence, with operating the magnetic field, the volume of blood flow can be controlled during the surgeries.

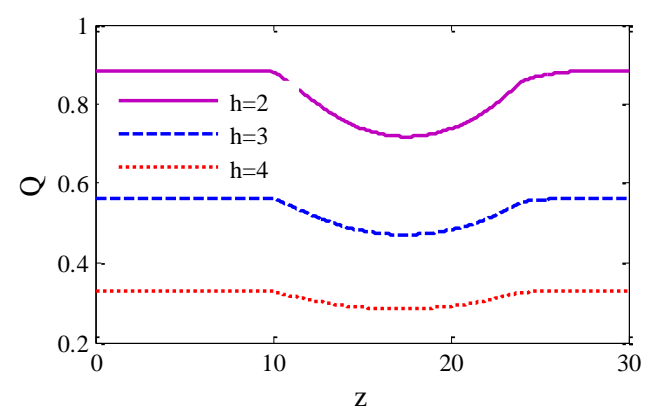

Figure 8. Distribution of the rate of flow for different values of Hartmann number

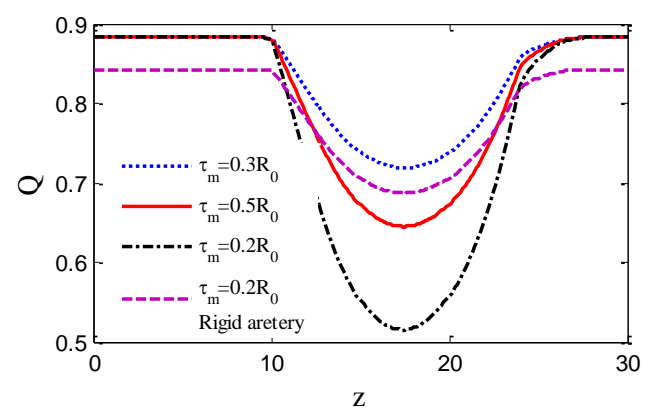

Figure 9. Distribution of the rate of flow for different stenosis sizes

Fig. 9 displays the comparison results regarding the volumetric flow rate through the elastic and rigid arteries, considering the evaluation of the effect of the stenosis size on the volumetric flow rate. It is clear that, the rate of flow decreases with the increase in the stenosis size and also, the flow rate through the elastic artery is less than the flow rate through the rigid artery at the same time.

The variation of the flow rate with time, for different amplitudes of body acceleration is illustrated in Fig 10. It is seen that the flow rate increases with the increase of amplitudes of body acceleration. Thus, the volumetric flow rate has an enhancing effect with its peak value of oscillation on the vibrating environmental system.

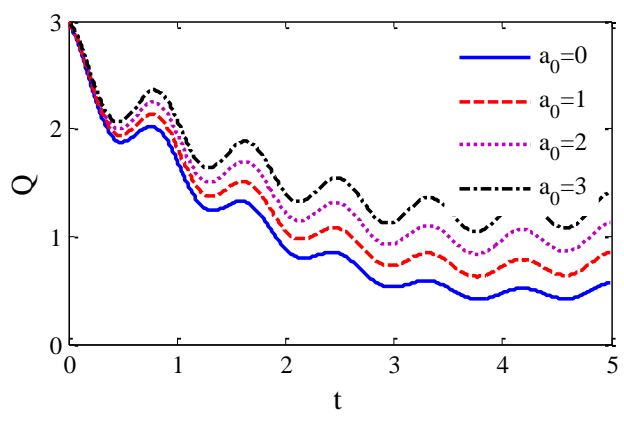

Figure 10. Distribution of volumetric flow rate with time for different amplitudes of body acceleration

The resistive impedance through a stenosed artery for different Hartmann numbers at $\mathrm{t}=4$ is presented in Fig 11. In this figure the resistive impedance increases with the increase in the Hartmann number. According to Eq. (20) the volumetric flow rate and the resistive impedance are inversely related, so unlike the volumetric flow rate, the resistive impedance increases with the increase in the Hartmann number.

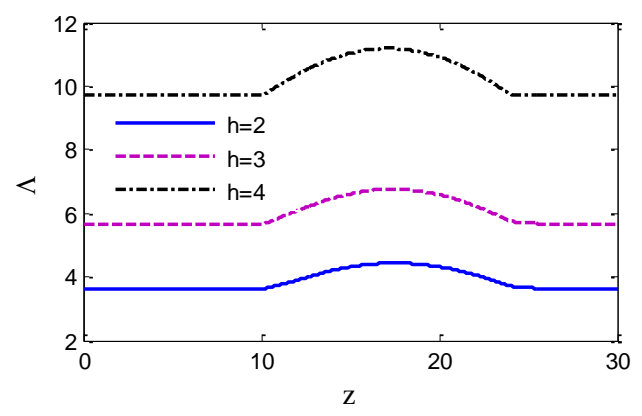

Figure 11. Distribution of the resistive impedance for different values of Hartmann number

\section{CONCLUSIONS}

In the present study, a two-dimensional, unsteady and laminar blood flow through a stenosed artery is studied. The governing nonlinear partial differential equations are solved numerically using the Crank-Nicolson scheme. Also, the influences of the effective parameters on the flow characteristics such as the velocity profile, temperature, the volumetric flow rate and the resistive to flow are examined. The present results demonstrate that the axial velocity and the flow rate increases with the increase in the stenosis size. The axial velocity has reducing effect on the magnetic field. For this reason, the flow rate and the resistive impedance are inversely related to enhancements in the Hartmann number. So, 
the volume of blood flow can be controlled during surgeries using a controlled magnetic field. The temperature decreases with the increase of the Prandtl number but the Nusselt number increases with the increase of the Prandtl number. The blood flow characteristics through elastic artery have been compared with the rigid one and this difference between the axial velocities shows the importance of applying the elastic assumption for the blood vessels.

\section{REFERENCES}

[1] Mortazavinia Z, Zare A, Mehdizadeh A. (2012). Effects of renal artery stenosis on realistic model of abdominal aorta and renal arteries incorporating fluid-structure interaction and pulsatile non-Newtonian blood flow. Applied Mathematics Mechanics 33(2): 165-176. http://dx.doi.org/10.1007/s10483-012-1541-6

[2] Hogan HA, Henriksen M. (1989). An evaluation of a micropolar model for blood flow through an idealized stenosis. Journal of Biomechanics 22(3): 21-218. http://dx.doi.org/10.1016/0021-9290(89)90089-4

[3] Chakravarty S, Mandal P. (2004). Unsteady flow of a two-layer blood stream past a tapered flexible artery under stenotic conditions. Computational Methods in Applied Mathematics 4(4): 391-409. http://dx.doi.org/10.2478/cmam-2004-0022

[4] Sankar D, Lee U. (2001). FDM analysis for MHD flow of a non-Newtonian fluid for blood flow in stenosed arteries. J. Mech. Sci. Technol. 25(10): 2573-2581. http://dx.doi.org/10.1007/s12206-011-0728-x

[5] Sankar D. (2011). Two-phase non-linear model for blood flowin asymmetric and axisymmetric stenosed arteries. Int. J. Non-Linear Mech 46(1): 296-305. https://doi.org/10.1016/j.ijnonlinmec.2010.09.011

[6] Srivastava VP, Rastogi R, Vishnoi R. (2010). A twolayered suspension blood flow through an overlapping stenosis. Computers \& Mathematics with Applications 60(3):

$432-441$ http://dx.doi.org/10.1016/j.camwa.2010.04.038

[7] Li L, Yan X, Yang J, Wang Q. (2017). Influence of defects on flow and mass transfer in the channel with micro-fluidic chips. Chemical Engineering Transactions 61: 1015-1020. https://doi.org/10.3303/CET1761167

[8] Lorenzini G, Casalena E. (2008). CFD analysis of pulsatile blood flow in an atherosclerotic human artery with eccentric plaques. Journal of Biomechanics 41(9): 1862-1870.

https://doi.org/10.1016/j.jbiomech.2008.04.009

[9] Dauyeshova B, Rojas-Solorzano L, Monaco E. (2017). Study of the motion of a droplet in a microchannel using Shan-Chen multiphase lattice Boltzmann model. Chemical Engineering Transactions 57: 1267-1272. https://doi.org/10.3303/CET1757212

[10] Mekheimer KS, El Kot M. (2008). The micropolar fluid model for blood flow through a tapered artery with a stenosis. Acta Mechanica Sinica 24(6): 637-644. http://dx.doi.org/10.1007/s10409-008-0185-7

[11] Sankar D. (2009). A two-fluid model for pulsatile flow in catheterized blood vessels. International Journal of Non-Linear Mechanics 44(4): 337-351. http://dx.doi.org/10.1016/j.ijnonlinmec.2008.12.008

[12] Srivastava V, Vishnoi R, Mishra S, Sinha P. (2001). A two-layered non-Newtonian arterial blood flows through an overlapping constriction. AAM: Int. J. 6(1): 41-57.

[13] Chakravarty S, Mandal P.K. (2000). Two-dimensional blood flow through tapered arteries under stenotic conditions. International Journal of Non-Linear Mechanics 35(5): 779-793. http://dx.doi.org/10.1016/S0020-7462(99)00059-1

[14] Ponalagusamy R, Tamil Selvi R. (2011). A study on twolayered model (Casson-Newtonian) for blood flow through an arterial stenosis: axially variable slip velocity at the wall. Journal of the Franklin Institute. 348(9): 2308-2321. http://dx.doi.org/10.1016/j.jfranklin.2011.06.020

[15] Haghighi AR, Kabdool AA, Asl MS, Kiyasatfar M. (2016). Numerical Investigation of Pulsatile Blood Flow in Stenosed Artery. International Journal of Applied and Computational Mathematics 2(4): 649-662. http://dx.doi.org/10.1007/s40819-015-0084-0

[16] Misra JC, Shit GC. (2009). Flow of a biomagnetic viscoelastic fluid in a channel with stretching walls. Journal of Applied Mechanics 76(6): 061006. https://doi.org/10.1115/1.3130448

[17] Misra JC, Shit GC, Chandra S, Kundu PK. (2011). Hydromagnetic flow and heat transfer of a second-grade viscoelastic fluid in a channel with oscillatory stretching walls: Application to the dynamics of blood flow. Journal of Engineering Mathematics 69(1): 91-100. http://dx.doi.org/10.1007/s10665-010-9376-x

[18] Misra JC, Sinha A, Shit GC. (2011). Mathematical modeling of blood flow in a porous vessel having double stenosis in the presence of an external magnetic field. International Journal of Biomathematics 4(02): 207-225. https://doi.org/10.1142/s1793524511001428

[19] Mekheimer KhS, Haroun MH, Elkot MA. (2011). Effects of magnetic field, porosity, and wall properties for anisotropically elastic multi-stenosis arteries on blood flow characteristics. Applied Mathematics and Mechanics 32(8): 1047-1064. http://dx.doi.org/10.1007/s10483-011-1480-7

[20] Shit GC. (2013). Computational modelling of blood flow development and its characteristics in magnetic environment. Modelling and Simulation in Engineering 12. https://doi.org/10.1155/2013/758748

[21] Alshare A, Tashtoush B, El-Khalil HH. (2013). Computational modelling of non-Newtonian blood flow through stenosed arteries in the presence of magnetic field. Journal of Biomechanical Engineering 135(11): 114503. https://doi.org/10.1115/1.4025107

[22] Shankar DS, Lee U. (2011). Nonlinear mathematical analysis for blood flow in a constricted artery under periodic body acceleration. Communications in Nonlinear Science and Numerical Simulation 16(11): 4390-4402. https://doi.org/10.1016/j.cnsns.2011.03.020

[23] Shit GC, Roy M. (2011). Pulsatile flow and heat transfer of a magneto-micropolar fluid through a stenosed artery under the influence of body acceleration. Journal of Mechanics in Medicine and Biology 11(03): 643-661. http://dx.doi.org/10.1142/S0219519411003909

[24] Haghighi AR, Asl MSH, Kiyasatfar M. (2015). Mathematical modeling of unsteady blood flow through elastic tapered artery with overlapping stenosed. Journal of the Brazilian Society of Mechanical Sciences and Engineering 37(2): 571-578 https://doi.org/10.1007/s40430-014-0206-3 
[25] Haghighi AR, Asl MSh. (2015). Mathematical modeling of micropolar fluid flow through an overlapping arterial stenosis. International Journal of Biomathematics, 8(04): 1550056. https://doi.org/10.1142/s1793524515500564

[26] Sankar D, Lee U. (2009). Mathematical modeling of pulsatile flow of non-Newtonian fluid in stenosed arteries. Communications in Nonlinear Science and Numerical Simulation 14(7): 2971-2981. http://dx.doi.org/10.1016/j.cnsns.2008.10.015

[27] Singh S. (2011). Numerical modeling of two-layered micropolar fluid through a normal and stenosed artery. IJE-Transactions A: Basics 24(2): 177-187.

[28] Shit GC, Majee S. (2015). Pulsatile flow of blood and heat transfer with variable viscosity under magnetic and vibration environment. Journal of Magnetism and Magnetic Materials 388: 106-115. http://dx.doi.org/10.1016/j.jmmm.2015.04.026

[29] Rafiee SE, Sadeghiazad MM. (2014). Three-dimensional and experimental investigation on the effect of cone length of throttle valve on thermal performance of a vortex tube using $\mathrm{k}-\varepsilon$ turbulence model. Applied Thermal Engineering 66(1-2): 65-74. http://dx.doi.org/10.1016/j.applthermaleng.2014.01.073

[30] Wang Y, Huang D. (2017). Effect of heat treatment temperature on the structure and tribological properties of nanometer lanthanum borate. International Journal of Heat and 53-8. http://dx.doi.org/10.18280/ijht.350107

[31] Asif M, Aftab H, Syed HA, Ali MA, Muizz PM. (2017). Simulation of corrugated plate heat exchanger for heat and flow analysis. International Journal of Heat and Technology 35(1): 205-210. http://dx.doi.org/10.18280/ijht.350127

[32] Mabood F, Ibrahim ShM, Lorenzini G, Lorenzin E. (2017). Radiation effects on Williamson nanofluid flow over a heated surface with magnetohydrodynamics. International Journal of Heat and Technology 35(1): 196204. http://dx.doi.org/10.18280/ijht.350126

[33] Haghighi AR, Asadi Chalak S. (2017). Mathematical modeling of blood flow through a stenosed artery under body acceleration. Journal of the Brazilian Society of Mechanical Sciences and Engineering (BMSE) 39(7): 2487-2494. http://dx.doi.org/10.1007/s40430-0170716-х

[34] Mukhopadhyay S, Layek GC. (2008). Numerical modeling of a stenosed artery using mathematical model of variable shape. Applications and Applied Mathematics: An International Journal 3(6): 308-328.

[35] Mandal P, Chakravarty S, Mandal A, Amin A. (2007). Effect of body acceleration on unsteady pulsatile flow of non-Newtonian fluid through a stenosed artery. Applied Mathematics and Computation. 189(1): 766-779. http://dx.doi.org/10.1016/j.amc.2006.11.139

[36] Belardinelli E, Cavalcanti S. (1991). A new nonlinear two-dimensional model of blood motion in tapered and elastic vessels. Computers in Biology and Medicine 21(1/2): 1-13. http://dx.doi.org/10.1016/00104825(91)90030-D

[37] Mustapha N, Amin N, Chakravarty S, Mandal P. (2009). Unsteady magnetohydrodynamic blood flow through irregular multi-stenosed arteries. Computers in Biology and Medicine 39(10): 896-906 http://dx.doi.org/10.1016/j.compbiomed.2009.07.004

\section{NOMENCLATURE}

$\mathrm{A}_{0}$

$\mathrm{A}_{1}$

d

$f_{p}$

$\mathrm{h}$

$\mathrm{k}_{\mathrm{r}}$

$\mathrm{L}$

$1_{0}$

$\mathrm{n} \geq 2$

$\mathrm{N}_{\mathrm{u}}$

$\mathrm{p}$

$P_{r}$

Q

$\mathrm{R}(\mathrm{z}, \mathrm{t})$

$\mathrm{R}_{0}$

$\mathrm{t}$

$\mathrm{u}$

$\mathrm{v}$

\section{Greek symbols}

$\alpha^{2}$
$\Delta \mathrm{t}$
$\Delta \mathrm{z}$
$\Delta \xi$
$\mu$
$\Lambda$
$\tau_{\mathrm{m}}$
$\varphi$

\section{Subscripts}

i

$\mathrm{j}$

$\mathrm{k}$
Constant amplitude of the pressure gradient

Amplitude of the pulsatile component

Upstream length

Pulse frequency

Hartmann number

Amplitude parameter

Finite length of the arterial segment

Length of the stenosis

parameter representing the asymmetry of the stenosis

Nusselt number

pressure

Prandtl number

Rate of flow

Radius of the arterial in the constricted

Radius of the nonstenotic

time

Axial velocity

Radial velocity

Womersley parameter
Time direction
Axial direction
Radial direction
Viscosity
Resistive impedance
Critical heigh
Phase angle

Phase angle 